\title{
Troublesome hydrates of alkaloids
}

\author{
D. E. Braun \\ Institute of Pharmacy, University of Innsbruck, Innrain 52c, 6020 Innsbruck, Austria \\ doris.braun@uibk.ac.at
}

Water plays a critical role in many steps of the pharmaceutical development as this small molecule has the ability to interact with compounds in numerous ways and may therefore significantly affect manufacturing processes and finally the quality of (pharmaceutical) products. The formation of a molecular compound (hydrate), where water becomes a part of the crystal lattice, is mostly accompanied with a significant change in the solid-state properties, and therefore this type of interaction must be seen as critical [1]. Hydrate formation itself is a widespread phenomenon and is known to occur for at least one third of drug molecules [2,3], and this trend is increasing significantly for new drug substances. Nevertheless, we are still not able to predict hydrates, their stability and dehydration mechanisms based on the molecular diagram only.

This talk will emphasise on the efforts that are sometimes required to identify solid forms of complex hydrate forming systems. Examples from our research will be used to illustrate how the combination of a variety of experimental techniques, covering temperature- and moisture-dependent stability, and computational modelling allows to generate sufficient kinetic, thermodynamic and structural information to understand hydrate formation and its impacts on relevant physicochemical properties.

The solid form landscape of brucine sulphate was elucidated, resulting in three hydrate forms and amorphous brucine sulphate. HyA was produced from water and the other two by dehydration starting from HyA. Removal of the essential water molecules stabilising the hydrate structures causes a collapse to the amorphous state [4]. Eight hydrate forms were verified for the related compound, strychnine sulphate. Three of the hydrates were found to be stable at ambient conditions. The other five hydrates are only observable at low(est) relative humidity $(\mathrm{RH})$ levels at room temperature. Some of the hydrates can only exist within a very narrow RH range and are therefore regarded as intermediate phases. The specific moisture and temperature conditions of none of the applied drying conditions yielded a crystalline water-free form, highlighting the essential role of water molecules for the formation and stability of crystalline strychnine sulphate [5].

Despite their structural similarity, marked differences in the formation of solid forms are seen for brucine and strychnine. One anhydrous form and 1,4-dioxane solvates were crystallized for strychnine, whereas two non-solvated polymorphs, four hydrates, an isostructural dehydrate, twelve solvates and two hetero-solvates are known to exist for brucine [6-8]. One of the brucine hydrates shows a non-stoichiometric (de)hydration behaviour, one collapses to an amorphous phase, and the third one to the polymorph which is stable at room temperature. Interestingly, each of the three hydrates may become the most stable form depending on temperature and water activity.

To conclude, this study demonstrates the importance of applying complimentary analytical techniques and appropriate approaches for understanding the stability ranges and transition behaviour between the solid forms of compounds with multiple hydrates.

[1] Khankari, R. K. \& Grant, D. J. W. (1995). Thermochim. Acta, 248, 61.

[2] Stahly, G. P. (2007). Cryst. Growth Des, 7, 1007.

[3] Reutzel-Edens, S. M., Braun D. E. \& Newman A. W. (2019). Polymorphism in the Pharmaceutical Industry: Solid Form and Drug Development, edited by R. Hilfiker \& M. Von Raumer: Wiley-VCH, pp. 159-188.

[4] Braun, D. E. (2020). CrystEngComm, 22, 7204.

[5] Braun, D. E., Gelbrich, T., Kahlenberg, V. \& Griesser, U. J. (2020). Cryst. Growth Des., $20,6069$.

[6] Braun, D. E. and Griesser, U. J. (2016). Cryst. Growth Des., 16, 6405.

[7] Braun, D. E. and Griesser, U. J. (2016). Cryst. Growth Des., 16, 6111.

[8] Watabe, T., Kobayashi, K., Hisaki, I., Tohnai, N. \& Miyata, M.Bull. (2007). Chem. Soc. Jpn., 80, 464.

\section{Keywords: hydrate; sulphate salts; brucine; strychnine; moisture (de)sorption; stability}

DEB gratefully acknowledges funding by the Elise Richter Programme of the Austrian Science Fund (FWF, project V436-N34). The computational results presented here have been achieved using the LEO HPC infrastructure of the University of Innsbruck.

Acta Cryst. (2021), A77, C205 